\title{
Effects of Anesthesia on Ozone-Induced Lung and Systemic Inflammation
}

\author{
Miranda L. Wilson ${ }^{1,3} \cdot$ Jarl A. Thysell ${ }^{1,2} \cdot$ Kristen K. Baumann $^{1} \cdot$ Danny V. Quaranta ${ }^{1,2} \cdot$ W. Sandy Liang ${ }^{1} \cdot$ \\ Michelle A. Erickson ${ }^{1,2}$ (D)
}

Received: 16 December 2021 / Accepted: 18 January 2022 / Published online: 23 February 2022

This is a U.S. government work and not under copyright protection in the U.S.; foreign copyright protection may apply 2022

\begin{abstract}
Purpose Anesthetics are required for procedures that deliver drugs/biologics, infectious/inflammatory agents, and toxicants directly to the lungs. However, the possible confounding effects of anesthesia on lung inflammation and injury are underreported. Here, we evaluated the effects of two commonly used anesthetic regimens on lung inflammatory responses to ozone in mice.

Methods We tested the effects of brief isoflurane (Iso) or ketamine/xylazine/atipamezole (K/X/A) anesthesia prior to ozone exposure (4 h, $3 \mathrm{ppm})$ on lung inflammatory responses in mice. Anesthesia regimens modeled those used for non-surgical intratracheal instillations and were administered $1-2 \mathrm{~h}$ or $24 \mathrm{~h}$ prior to initiating ozone exposure.

Results We found that Iso given 1-2 h prior to ozone inhibited inflammatory responses in the lung, and this effect was absent when Iso was given $23-24 \mathrm{~h}$ prior to ozone. In contrast, $\mathrm{K} / \mathrm{X} / \mathrm{A}$ given $1-2 \mathrm{~h}$ prior to ozone increased lung and systemic inflammation.

Conclusion Our results highlight the need to comprehensively evaluate anesthesia as an experimental variable in the assessment of lung inflammation in response to ozone and other inflammatory stimuli.
\end{abstract}

Keywords Ozone $\cdot$ Inflammation $\cdot$ Isoflurane $\cdot$ Ketamine $\cdot$ Xylazine $\cdot$ Atipamezole $\cdot$ Serum amyloid A

\section{Introduction}

Ozone $\left(\mathrm{O}_{3}\right)$ is a widespread air toxicant with well-established inflammatory effects in the lungs, but it also affects other organs, such as the liver, spleen, and brain. Approaches for selectively inhibiting lung inflammation may provide insight on the systemic effects of $\mathrm{O}_{3}$ and could involve direct delivery of substances to the lungs through the trachea prior to $\mathrm{O}_{3}$ exposure. Volatile anesthetics such as

Michelle A. Erickson

mericks9@uw.edu

1 Veterans Administration Puget Sound Healthcare System, 1660 S. Columbian Way, S-182, Seattle, WA 98108, USA

2 Division of Gerontology and Geriatric Medicine, Department of Medicine, University of Washington, 325 th Avenue, Box 359755, Seattle, WA 98104, USA

3 Present Address: Department of Cell, Developmental, and Regenerative Biology, Icahn School of Medicine at Mount Sinai, 1 Gustave L. Levy Pl, New York, NY 10029, USA isoflurane are frequently used for intratracheal instillations and other surgeries [1-3]. However, isoflurane can have anti-inflammatory effects $[4,5]$, which may be most potent in the respiratory tract since it is the first site of exposure. Injectable anesthetics such as ketamine/xylazine are also used for intratracheal instillations [6], but may also modulate inflammatory responses [7-10]. Currently, little is known about how anesthesia prior to $\mathrm{O}_{3}$ exposure affects inflammatory responses. To test this, we exposed mice to isoflurane (Iso) or ketamine/xylazine followed by atipamezole reversal $(\mathrm{K} / \mathrm{X} / \mathrm{A})$ prior to $\mathrm{O}_{3}$. Conditions of anesthesia were designed to replicate those used for non-surgical intratracheal instillations of substances such as drugs or biologics that modulate effects of $\mathrm{O}_{3}$ on the lungs in mice. The day after $\mathrm{O}_{3}$ exposure, mice were evaluated for markers of systemic and pulmonary inflammation. We found that Iso and K/X/A modify lung inflammation in response to $\mathrm{O}_{3}$. 


\section{Methods}

\section{Animal Use}

Female CD-1 mice, age 10-12 weeks, (Charles River Laboratories, Malvern, PA, USA) were used for air and $\mathrm{O}_{3}$ exposures. Females were used because they have more robust inflammatory responses to $\mathrm{O}_{3}$. Mice were given food and water ad libitum and kept on a 12-h light/12-h dark cycle. Protocols were approved by the institutional animal care and use committee of the VA Puget Sound Healthcare System.

\section{Anesthesia}

Isoflurane was administered by first weighing all mice, followed by placing mice in an induction chamber filled with $4 \%$ isoflurane for $1.5 \mathrm{~min}$ and then transferring to a nose cone where $3 \%$ isoflurane was used to maintain deep anesthesia for $5 \mathrm{~min}$, which approximates the maximum time that a skilled technician needs to complete an intratracheal injection. Control mice were placed in the empty anesthesia chamber with room air for $1.5 \mathrm{~min}$ and then returned to their home cage. 1-2 h or 23-24 h after isoflurane exposure, $\mathrm{O}_{3}$ exposures began. Ketamine $(80 \mathrm{mg} / \mathrm{kg})$ and xylazine
$(10 \mathrm{mg} / \mathrm{kg})$ in sterile saline were administered by intraperitoneal injection (IP). 20-30 min after the injection, mice were given $1 \mathrm{mg} / \mathrm{kg}$ atipamezole IP to quickly counteract the effects of the anesthesia. Control mice were given IP saline. $1-2 \mathrm{~h}$ after the atipamezole injection, $\mathrm{O}_{3}$ exposures began. All mice were numbered with a marker to track their identity, and mice were randomized so that the average time between anesthesia and air/ozone exposure was equivalent between groups. A schematic of $\mathrm{O}_{3}$ and anesthesia regimens is shown in Fig. 1.

\section{$\mathrm{O}_{3}$ Exposure}

$\mathrm{O}_{3}$ exposures were conducted at $3 \mathrm{ppm}$ for $4 \mathrm{~h}$ (10:00-14:00), which induce a robust inflammatory response without inducing respiratory distress [11]. For the exposure duration, 3-4 mice were housed in standard mouse cages with free access to water, but without food or bedding to prevent consumption of ozonated food or bedding materials. Up to four cages at a time were placed in a $30^{\prime \prime} \times 20^{\prime \prime} \times 20^{\prime \prime}$ polypropylene chamber where $\mathrm{O}_{3}$ (3 ppm, chamber 1) or compressed dry air (chamber 2) was pumped into the chamber at equivalent rates. Anesthesia and $\mathrm{O}_{3}$ exposures were replicated at least once to capture day-today variability, and results for each dose showed consistent trends. $\mathrm{O}_{3}$ levels in the chambers were generated and regulated

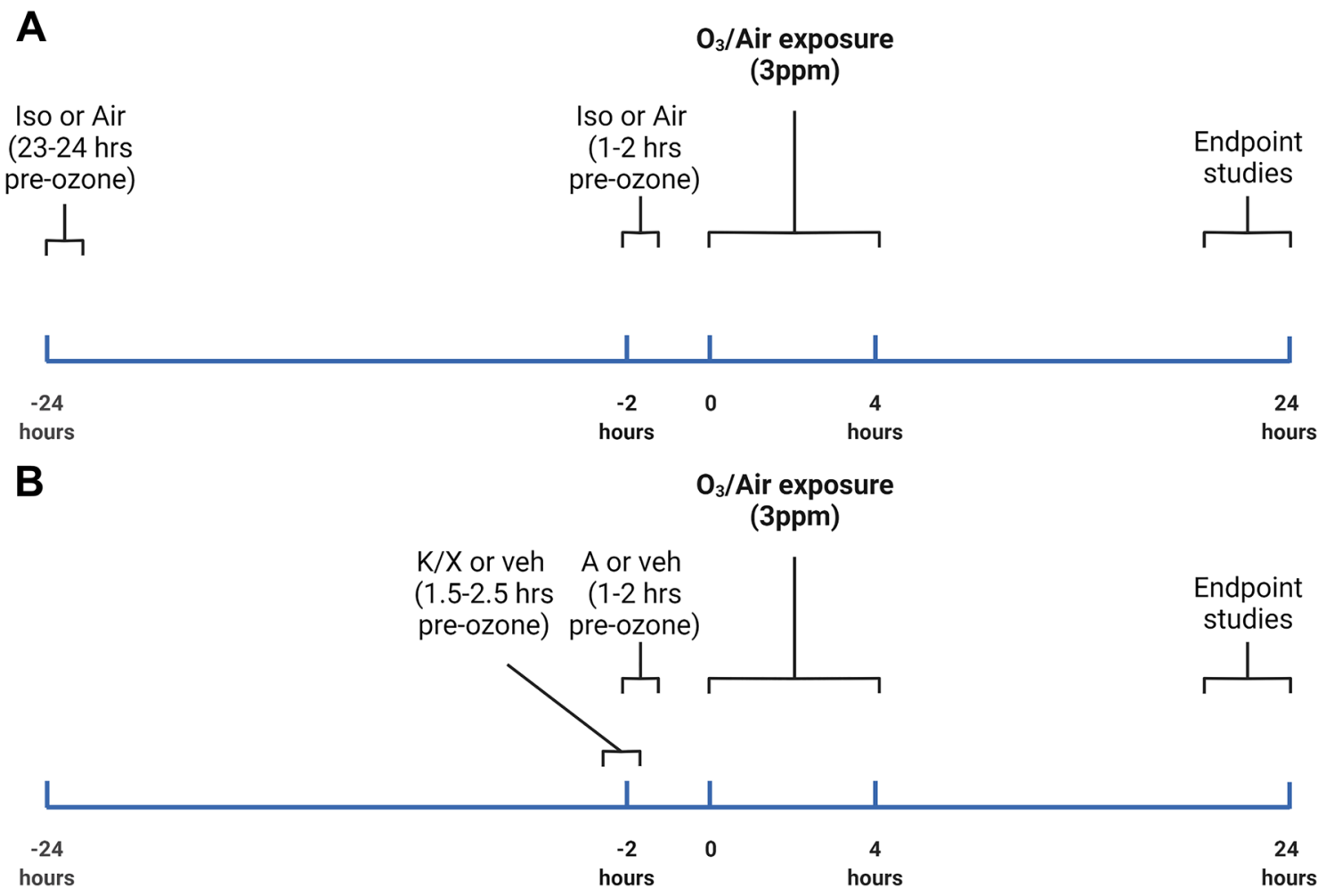

Fig. 1 Schematic of anesthesia/ozone $\left(\mathrm{O}_{3}\right)$ exposure protocols. A shows the exposure protocol for mice pre-exposed to isoflurane (Iso) or air control, and $\mathbf{B}$ shows the exposure protocol for mice pre- exposed to Ketamine/Xylazine (K/X) and atipamezole (A) or saline vehicle. Created with Biorender.com 
using an Oxycycler AT42 system (BioSpherix, Parish NY, USA). Prior to each experiment, the system was calibrated using a model 106-L O3 detector (2B Technologies, Boulder, $\mathrm{CO}, \mathrm{USA}$ ), and $\mathrm{O}_{3}$ levels were recorded from an inlet valve in one of the $\mathrm{O}_{3}$-exposed mouse cages every $10 \mathrm{~s}$ for the duration of exposures. In all experiments, $\mathrm{O}_{3}$ achieved its target concentration within $10 \mathrm{~min}$, and levels were regulated within $10 \%$ of the target concentration ( $3 \mathrm{ppm} \pm 0.3 \mathrm{ppm})$ thereafter.

\section{Blood Collection and Serum Amyloid A Measurement}

22-24 h after the start of $\mathrm{O}_{3}$ exposure (12:00-14:00), mice were re-weighed and deeply anesthetized with IP urethane (Millipore Sigma, St. Louis, MO, USA). Blood was collected from the abdominal aorta, allowed to clot for $30 \mathrm{~min}$, and then placed on ice. Blood was centrifuged at $2500 \times g$ for $15 \mathrm{~min}$ and serum was collected, aliquoted, and frozen at $-80{ }^{\circ} \mathrm{C}$. Serum was diluted 1/10,000 for mice exposed to ozone and 1/50 for mice not exposed to ozone. Serum amyloid A (SAA) mouse duoset kits ( $\mathrm{R}$ and $\mathrm{D}$ systems, Minneapolis, MN, USA) were used to quantify SAA in serum.

\section{WLL and Pulmonary Inflammation Assessment}

Whole-lung lavage (WLL) was performed on mice by perfusing and aspirating the lungs through the trachea three times with $1 \mathrm{~mL}$ sterile phosphate-buffered saline ( $3 \mathrm{~mL}$ total). WLL fluid was stored on ice and centrifuged at $200 \times g$ for $5 \mathrm{~min}$ at 4 ${ }^{\circ} \mathrm{C}$. The supernatant was removed and $0.5 \mathrm{mLs}$ of supernatant were reserved to resuspend the cell pellet. The remainder was frozen for measurement of total WLLL protein, which was performed using a microBradford assay. Total cells were manually counted using a hemacytometer. Differential cell counts were performed on Hemacolor-stained cytocentrifuge preparations. Cell counts were performed using ImageJ, and at least 200 cells were counted to determine relative cell proportions.

\section{Statistics}

Statistical analysis was done with Prism 8.4.2 (GraphPad Software, San Diego, CA, USA). Data are reported as mean \pm SD and analyzed by two-way ANOVA for main effects and interactions and Tukey's multiple comparisons test for comparisons of group means.

\section{Results}

\section{Effects of Isoflurane and Ketamine/Xylazine/ Atipamezole Anesthesia on Ozone-Induced Pulmonary Inflammation and Injury}

We first determined whether $\mathrm{O}_{3}$-induced responses in the lungs differed with Iso or K/X/A. Since drugs or other interventions to be administered intratracheally would ideally be given a short time before exposure to ozone to maximize their effects/minimize their metabolism, we evaluated anesthesia administration at $1-2 \mathrm{~h}$ prior to ozone exposure. We chose 1-2 $\mathrm{h}$ because this time frame allows the mice to recover from anesthesia and also reflects a time window that is typically sufficient for an administered drug to sufficiently activate/inhibit its biological target. A pilot study indicated that Iso given $1-2 \mathrm{~h}$ before $\mathrm{O}_{3}$ inhibited inflammation, suggesting that this regimen is unsuitable for studying anti-inflammatory interventions for $\mathrm{O}_{3}$ exposure. Therefore, we included a 24-h Iso pre-exposure in experimental replicates to determine whether the antiinflammatory effects of Iso were sustained. The 24-h time point was chosen because the Iso and $\mathrm{O}_{3}$ exposures could then occur at approximately the same time of day as the 1-2-h Iso pre-exposure group. We compared K/X/A to Iso $1-2-\mathrm{h}$ pre $-\mathrm{O}_{3}$ exposure to determine whether $\mathrm{K} / \mathrm{X} / \mathrm{A}$ is a suitable alternative anesthesia regimen to Iso (Schematics in Fig. 1A, B). Although the controls for ketamine were IP injected with saline, whereas Iso controls were not, we found that IP injections did not significantly influence the measured outcomes, and so we combined the Iso and Ketamine no anesthesia control groups for analysis. $\mathrm{O}_{3}$ significantly increased WLL total cells, macrophages, and neutrophils except in the 1-2-h Iso group (Fig. 2A-C). We further show that mice exposed to K/X/A and $\mathrm{O}_{3}$ had significantly increased WLL total cells, macrophages, and neutrophils vs. other $\mathrm{O}_{3}$-exposed groups. $\mathrm{O}_{3}$ significantly increased WLL protein in the no anesthesia group and 23-24-h Iso groups, but not in the 1-2-h Iso or K/X/A groups (Fig. 2D). The $F$-statistics and $p$-values for main effects and interactions are reported in Table 1.

\section{Effects of Iso and K/X/A Anesthesia on Ozone-Induced Systemic Inflammation and Weight Loss}

We next determined whether the effects of anesthesia extended to markers of ozone-induced systemic inflammation and behavioral effects. We have previously shown that the acute-phase protein SAA is consistently increased in blood following a $3 \mathrm{ppm}$ exposure to $\mathrm{O}_{3}$ and 


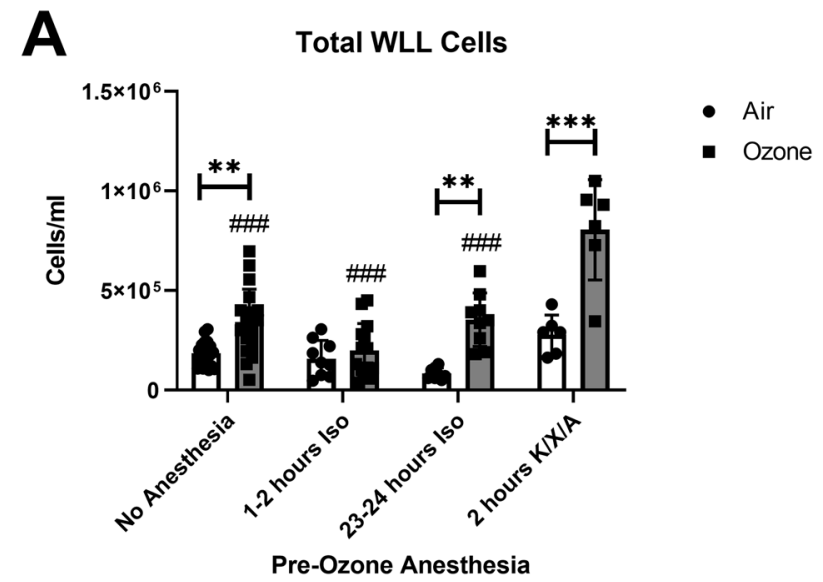

C
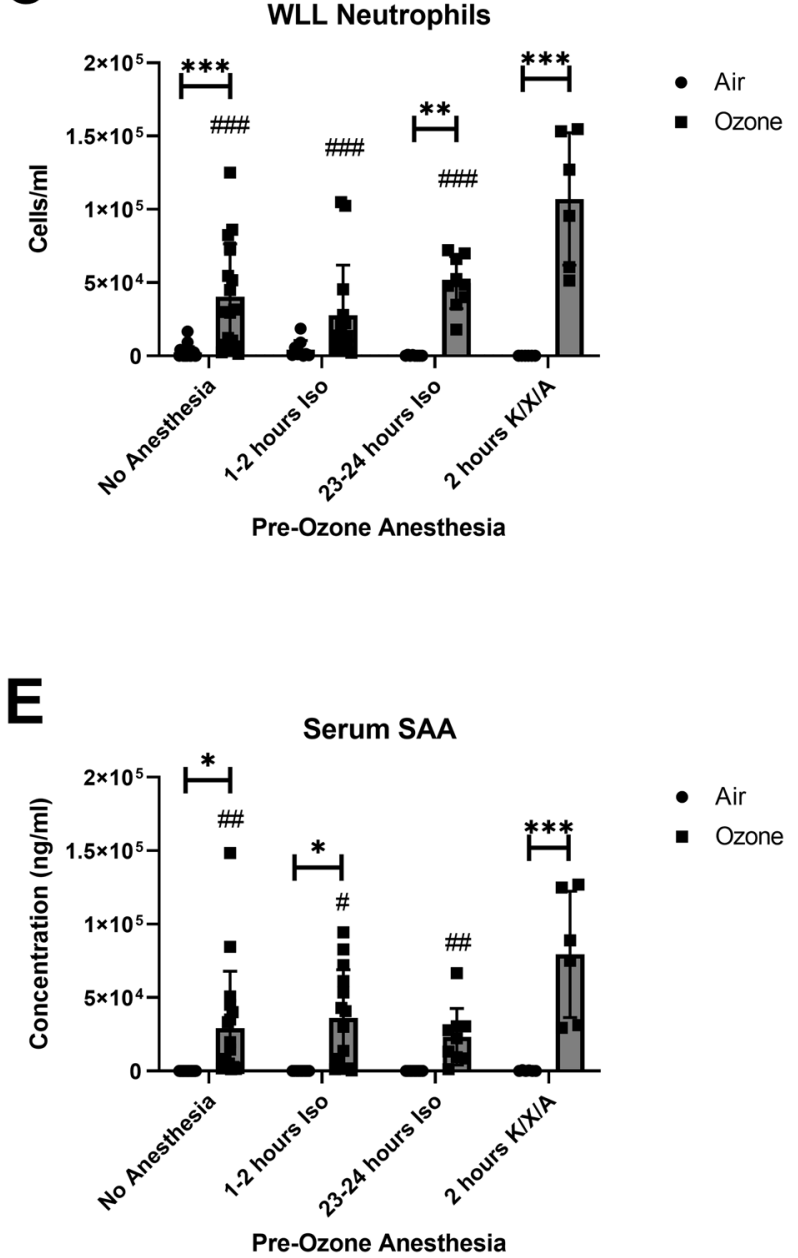

Fig. 2 Effects of isoflurane (Iso) and ketamine/xylazine/atipamezole $(\mathrm{K} / \mathrm{X} / \mathrm{A})$ on inflammatory $(\mathbf{A}-\mathbf{C})$ and vascular damage (D) markers in the lung, blood levels of serum amyloid A (SAA, E), and weight loss $(\mathbf{F})$. In all panels, $n=6-20$ per group. $* * * p<0.001$,
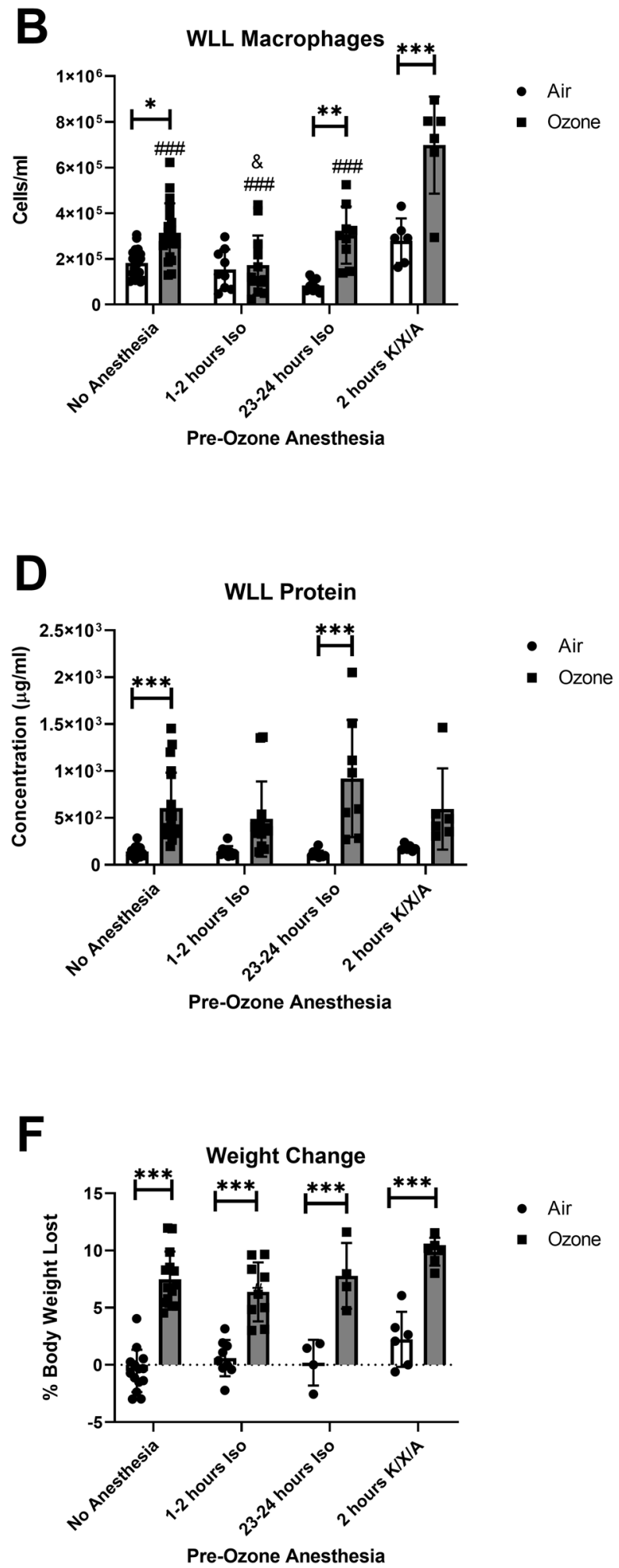

${ }^{*} * p<0.01,{ }^{*} p<0.05$, air vs. O3. ${ }^{\# \# \#} p<0.001,{ }^{\# \#} p<0.01,{ }^{\#} p<0.05$, vs. $\mathrm{K} / \mathrm{X} / \mathrm{A}+\mathrm{O}_{3}$ group. ${ }^{\&} p<0.05$ vs. No Anesthesia $+\mathrm{O}_{3}$. Group mean comparisons were carried out using Tukey's multiple comparisons test 
Table 1 Two-way ANOVA analysis results showing percentages of variation by factor (main effects, interactions) and $F$-statistics

\begin{tabular}{llll}
\hline Figure & Anesthesia & Exposure & Interaction \\
\hline 2A-total WLL cells & $28.97 \% ; F(3,82)=22.15, p<0.0001$ & $29.96 \% ; F(1,82)=68.73, p<0.0001$ & $12.79 \% ; F(3,82)=9.780, p<0.0001$ \\
2B-WLL macrophages & $31.77 \% ; F(3,82)=23.49, p<0.0001$ & $25.59 \% ; F(1,82)=56.74, p<0.0001$ & $11.72 \% ; F(3,82)=8.662, p<0.0001$ \\
2C-WLL neutrophils & $9.766 \%, F(3,82)=6.535, p=0.0005$ & $44.88 \% ; F(1,82)=90.08, p<0.0001$ & $11.82 \% ; F(3,82)=7.907, p=0.0001$ \\
2D-WLL total protein & $2.776 \% ; F(3,81)=1.309, p=0.2773$ & $33.06 \% ; F(1,81)=46.76, p<0.0001$ & $3.888 \% ; F(3,81)=1.833, p=0.1478$ \\
2E-serum SAA & $7.296 \%, F(3,81)=3.668, p=0.0156$ & $35.35 \% ; F(1,81)=53.31, p<0.0001$ & $7.204 \% ; F(3,81)=3.622, p=0.0165$ \\
2F-weight change & $7.784 \% ; F(3,58)=6.412, p=0.0008$ & $52.74 \% ; F(1,58)=130.3, p<0.0001$ & $1.903 \% ; F(3,58)=1.567, p=0.2070$ \\
\hline
\end{tabular}

For Fig. 2D, one sample was excluded because of apparent blood contamination. For Fig. 2E, one sample was excluded because not enough serum was recovered to complete the assay. For Fig. 2 F, body weights were not measured in one experimental replicate and so 23 samples are missing

its upregulation occurs in the absence of changes in other pro-inflammatory cytokines in blood [12]. Weight loss is a measurable outcome of sickness behavior, which can be induced by $\mathrm{O}_{3}$. Anesthesia has no effect on $\mathrm{O}_{3}$-induced weight loss based on the post hoc mean comparisons of the ozone groups (Fig. 2F); however, there was a significant main effect of treatment that explained $5.172 \%$ of the total variation (Table 1 ). $\mathrm{O}_{3}$ significantly increased SAA levels in all groups except for mice exposed to Iso 23-24 h prior (Fig. 2E). SAA levels induced by $\mathrm{O}_{3}$ were significantly higher in mice pre-anesthetized with K/X/A vs no anesthesia or Iso.

\section{Discussion}

We found that isoflurane inhibits $\mathrm{O}_{3}$-induced pulmonary inflammation if given $1-2 \mathrm{~h}$ prior to exposure, and this effect diminishes by $24 \mathrm{~h}$. Isoflurane induces anesthesia via potentiation of inhibitory $\gamma$-aminobutyric acid type A (GABA A) receptors and inhibition of glutamatergic $\alpha$-amino-3-hydroxy-5-methyl-4-isoxazolepropionic acid (AMPA) and $N$-methyl-d-aspartate (NMDA) receptors [13]. Isoflurane also has anti-inflammatory activities in a variety of rodent models [4], although the mechanisms are not well defined. It has been shown that activation of GABA A receptors and inhibition of NMDA receptors have anti-inflammatory effects on immune cells [14-16], suggesting that the anti-inflammatory activities of Iso could be mediated through direct actions on leukocytes. In contrast to Iso, $\mathrm{K} / \mathrm{X} / \mathrm{A}$ increased $\mathrm{O}_{3}$-induced inflammatory responses to $\mathrm{O}_{3}$. Anti-inflammatory/protective effects through NMDA receptor antagonism have been described for ketamine and ketamine/xylazine combinations [9]. Whereas xylazine is a $\alpha 2$-adrenergic receptor agonist and potentiates anesthesia, atipamezole is an antagonist and leads to rapid anesthesia reversal within about 20 min [17]. The data on pro- vs. anti-inflammatory activities of the $\alpha 2$ adrenergic receptor are equivocal; however, a recent study showed that atipamezole may promote or prolong inflammation through its action via a $\alpha 2$-independent mechanism [18]. It is also possible that anesthetics affect the amount of leukocyte trafficking to the lungs by altering the cytokine and chemokine profiles that are induced by $\mathrm{O}_{3}$. Acute exposures to $\mathrm{O}_{3}$ upregulate cytokines and chemokines such as CCL11, IL-6, G-CSF, CXCL10, and CXCL1 in a dose-dependent and cell type-specific manner [19]. Cytokines, such as TNF- $\alpha$, IL-1, and IL-6, chemokines, such as CXCL1, CXCL10, and CCL7, and chemokine receptors such as CCR2 have been shown to contribute to leukocyte trafficking to the lungs in response to $\mathrm{O}_{3}$ [20-25]. Future work is needed to delineate the immunomodulatory mechanisms of Iso and $\mathrm{K} / \mathrm{X} / \mathrm{A}$.

Our results are the first to show that isoflurane exposure prior to $\mathrm{O}_{3}$ inhibits pulmonary inflammation, whereas K/X/A exposure has an enhancing effect. These results complement other recent works that have investigated anesthesia's effects on pulmonary inflammation in response to ozone and other pro-inflammatory agents [5, $16,26]$ and effects of anesthetics on the recovery of cells from WLL [27]. Although the scope of our study is limited in that we did not define a full-time course of anesthesia's effects, our results highlight that brief, acute anesthetic regimens can alter inflammatory responses to $\mathrm{O}_{3}$. We conclude that anesthetics are important experimental variables whose evaluation is necessary for studies requiring their use prior to exposure to $\mathrm{O}_{3}$ or other pathological stimuli.

Author Contributions All authors contributed to the collection and analysis of data, editing of manuscript drafts, and approved the final version of the manuscript. MAE, JAT, KKB, and MLW contributed to the study conception and design and manuscript preparation.

Funding This study was funded by the National Institutes of Health (R21ES029657, MAE) and the Veterans Administration (MAE). 


\section{Declarations}

Conflict of interest The authors have no relevant financial or non-financial interests to disclose.

Ethical Approval The use of mice on this study was approved by the VA Puget Sound Healthcare System IACUC, protocol 0924. Human subjects were not used on this study.

Open Access This article is licensed under a Creative Commons Attribution 4.0 International License, which permits use, sharing, adaptation, distribution and reproduction in any medium or format, as long as you give appropriate credit to the original author(s) and the source, provide a link to the Creative Commons licence, and indicate if changes were made. The images or other third party material in this article are included in the article's Creative Commons licence, unless indicated otherwise in a credit line to the material. If material is not included in the article's Creative Commons licence and your intended use is not permitted by statutory regulation or exceeds the permitted use, you will need to obtain permission directly from the copyright holder. To view a copy of this licence, visit http://creativecommons.org/licenses/by/4.0/.

\section{References}

1. Driscoll KE et al (2000) Intratracheal instillation as an exposure technique for the evaluation of respiratory tract toxicity: uses and limitations. Toxicol Sci 55:24-35. https://doi.org/10.1093/toxsci/ 55.1 .24

2. Morales-Nebreda L et al (2014) Intratracheal administration of influenza virus is superior to intranasal administration as a model of acute lung injury. J Virol Methods 209:116-120. https://doi. org/10.1016/j.jviromet.2014.09.004

3. Lawrenz MB, Fodah RA, Gutierrez MG, Warawa J (2014) Intubation-mediated intratracheal (IMIT) instillation: a noninvasive, lung-specific delivery system. J Vis Exp. https://doi.org/10.3791/ 52261

4. Lee YM, Song BC, Yeum KJ (2015) Impact of volatile anesthetics on oxidative stress and inflammation. Biomed Res Int 2015:242709. https://doi.org/10.1155/2015/242709

5. Lacher SE, Johnson C, Jessop F, Holian A, Migliaccio CT (2010) Murine pulmonary inflammation model: a comparative study of anesthesia and instillation methods. Inhalation Toxicol 22:77-83. https://doi.org/10.3109/08958370902929969

6. Helms MN, Torres-Gonzalez E, Goodson P, Rojas M (2010) Direct tracheal instillation of solutes into mouse lung. J Vis Exp. https://doi.org/10.3791/1941

7. Tan S, Wang Y, Chen K, Long Z, Zou J (2017) Ketamine alleviates depressive-like behaviors via down-regulating inflammatory cytokines induced by chronic restraint stress in mice. Biol Pharm Bull 40:1260-1267. https://doi.org/10.1248/bpb.b17-00131

8. Chen MH et al (2018) Rapid inflammation modulation and antidepressant efficacy of a low-dose ketamine infusion in treatmentresistant depression: a randomized, double-blind control study. Psychiatry Res 269:207-211. https://doi.org/10.1016/j.psychres. 2018.08.078

9. Erdem MK, Yurdakan G, Yilmaz-Sipahi E (2014) The effects of ketamine, midazolam and ketamine/xylazine on acute lung injury induced by alpha-naphthylthiourea in rats. Adv Clin Exp Med 23:343-351. https://doi.org/10.17219/acem/37124

10. Szelenyi $\mathbf{J}$ et al (2000) Opposite role of alpha2- and betaadrenoceptors in the modulation of interleukin-10 production in endotoxaemic mice. NeuroReport 11:3565-3568. https://doi.org/ 10.1097/00001756-200011090-00032

11. Kierstein $\mathrm{S}$ et al (2008) Ozone inhalation induces exacerbation of eosinophilic airway inflammation and hyperresponsiveness in allergen-sensitized mice. Allergy 63:438-446. https://doi.org/10. 1111/j.1398-9995.2007.01587.x

12. Erickson MA et al (2017) Serum amyloid A: an ozone-induced circulating factor with potentially important functions in the lungbrain axis. FASEB J 31:3950-3965. https://doi.org/10.1096/fj. 201600857RRR

13. Michelson NJ, Kozai TDY (2018) Isoflurane and ketamine differentially influence spontaneous and evoked laminar electrophysiology in mouse V1. J Neurophysiol 120:2232-2245. https://doi.org/ $10.1152 /$ jn.00299.2018

14. Reyes-Garcia MG, Hernandez-Hernandez F, Hernandez-Tellez B, Garcia-Tamayo F (2007) GABA (A) receptor subunits RNA expression in mice peritoneal macrophages modulate their IL-6/ IL-12 production. J Neuroimmunol 188:64-68. https://doi.org/10. 1016/j.jneuroim.2007.05.013

15. Bhandage AK et al (2018) GABA regulates release of inflammatory cytokines from peripheral blood mononuclear cells and $\mathrm{CD} 4(+) \mathrm{T}$ cells and is immunosuppressive in type 1 diabetes. EBioMedicine 30:283-294. https://doi.org/10.1016/j.ebiom.2018. 03.019

16. Wang $\mathrm{Y}$ et al (2016) N-methyl-D-aspartate receptor activation mediates lung fibroblast proliferation and differentiation in hyperoxia-induced chronic lung disease in newborn rats. Respir Res 17:136. https://doi.org/10.1186/s12931-016-0453-1

17. Mees L et al (2018) Faster emergence behavior from ketamine/ xylazine anesthesia with atipamezole versus yohimbine. PLoS ONE 13:e0199087. https://doi.org/10.1371/journal.pone.0199087

18. Hu J et al (2018) Dexmedetomidine prevents cognitive decline by enhancing resolution of high mobility group box 1 proteininduced inflammation through a vagomimetic action in mice. Anesthesiology 128:921-931. https://doi.org/10.1097/ALN.00000 00000002038

19. Tovar A et al (2020) Transcriptional profiling of the murine airway response to acute ozone exposure. Toxicol Sci 173:114-130. https://doi.org/10.1093/toxsci/kfz219

20. Bhalla DK, Reinhart PG, Bai C, Gupta SK (2002) Amelioration of ozone-induced lung injury by anti-tumor necrosis factor-alpha. Toxicol Sci 69:400-408. https://doi.org/10.1093/toxsci/69.2.400

21. Cho HY, Zhang LY, Kleeberger SR (2001) Ozone-induced lung inflammation and hyperreactivity are mediated via tumor necrosis factor-alpha receptors. Am J Physiol Lung Cell Mol Physiol 280:L537-546. https://doi.org/10.1152/ajplung.2001.280.3.L537

22. Johnston RA, Schwartzman IN, Flynt L, Shore SA (2005) Role of interleukin-6 in murine airway responses to ozone. Am J Physiol Lung Cell Mol Physiol 288:L390-397. https://doi.org/10.1152/ ajplung.00007.2004

23. Park JW et al (2004) Interleukin-1 receptor antagonist attenuates airway hyperresponsiveness following exposure to ozone. Am J Respir Cell Mol Biol 30:830-836. https://doi.org/10.1165/rcmb. 2003-0373OC

24. Michalec L et al (2002) CCL7 and CXCL10 orchestrate oxidative stress-induced neutrophilic lung inflammation. J Immunol 168:846-852. https://doi.org/10.4049/jimmunol.168.2.846

25. Francis $\mathrm{M}$ et al (2017) Editor's highlight: CCR2 regulates inflammatory cell accumulation in the lung and tissue injury following ozone exposure. Toxicol Sci 155:474-484. https://doi.org/10. 1093/toxsci/kfw226

26. Yuki K et al (2021) Anesthetics isoflurane and sevoflurane attenuate flagellin-mediated inflammation in the lung. Biochem Biophys 
Res Commun 557:254-260. https://doi.org/10.1016/j.bbrc.2021. 04.045

27. Tighe RM, Birukova A, Yaeger MJ, Reece SW, Gowdy KM (2018) Euthanasia- and lavage-mediated effects on bronchoalveolar measures of lung injury and inflammation. Am J Respir Cell Mol Biol 59:257-266. https://doi.org/10.1165/rcmb.2017-0357OC
Publisher's Note Springer Nature remains neutral with regard to jurisdictional claims in published maps and institutional affiliations. 